\title{
Why Are Some Enzymes Dimers? Flexibility and Catalysis in Thermotoga maritima Dihydrofolate Reductase
}

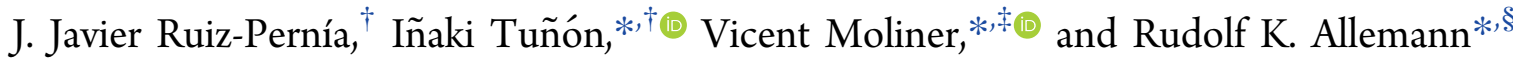 \\ ${ }^{\dagger}$ Departamento de Química Física, Universitat de Valencia, 46100 Burjassot, Valencia, Spain \\ ${ }^{\ddagger}$ Departamento de Química Física y Analítica, Universitat Jaume I, 12071 Castellón, Spain \\ ${ }^{\S}$ School of Chemistry, Cardiff University, Main Building, Park Place, Cardiff CF10 3AT, United Kingdom
}

\section{Supporting Information}

ABSTRACT: Dihydrofolate reductase from Thermotoga maritima (TmDFHFR) is a dimeric thermophilic enzyme that catalyzes the hydride transfer from the cofactor NADPH to dihydrofolate less efficiently than other DHFR enzymes, such as the mesophilic analogue Escherichia coli DHFR (EcDHFR). Using QM/MM potentials, we show that the reduced catalytic efficiency of TmDHFR is most likely due to differences in the amino acid sequence that stabilize the M20 loop in an open conformation, which prevents the formation of some interactions in the transition state and increases the number of water molecules in the active site. However, dimerization provides two advantages to the thermophilic enzyme: it protects its structure against denaturation by reducing thermal fluctuations and it provides a less negative activation entropy, toning down the increase of the activation free energy with temperature. Our molecular picture is confirmed by the analysis of the temperature dependence of enzyme kinetic isotope effects in different DHFR enzymes.

KEYWORDS: Dihydrofolate Reductase, enzyme dimers, QM/MM methods, free energy calculations, enzyme kinetic isotope effects

\section{INTRODUCTION}

Detailed knowledge of the mechanisms employed by enzymes to speed up chemical processes can serve as a guide for the design of novel biocatalysts. ${ }^{1}$ However, many of the factors that govern the behavior of these amazing catalysts, including the impact of their protein quaternary structure on catalysis, remain poorly understood. Comparative studies of dihydrofolate reductase (DHFR) homologues offer a unique opportunity to elucidate the impact of protein dimerization on the chemical step. $^{2,3}$ DHFRs catalyze the chemical transformation of dihydrofolate $\left(\mathrm{H}_{2} \mathrm{~F}\right)$ to tetrahydrofolate $\left(\mathrm{H}_{4} \mathrm{~F}\right)$ and due to their crucial role in protein and DNA synthesis are found in organisms across all kingdoms of life. $\mathrm{H}_{4} \mathrm{~F}$ is the reduced form of folic acid and is the primary carrier of $\mathrm{C} 1$ units in many important cellular processes. It is the precursor of derivatives that participate in a myriad of biosynthetic reactions. ${ }^{4}$ The reaction involves hydride transfer from $\mathrm{C} 4$ of $\mathrm{NADPH}$ to $\mathrm{C} 6$ of $\mathrm{H}_{2} \mathrm{~F}$ after a solvent-assisted protonation of $\mathrm{N} 5$ of $\mathrm{H}_{2} \mathrm{~F}$ (see Scheme 1). ${ }^{5}$

DHFRs of different organisms share important parts of their sequences and most of the residues of their active site are identical. Nevertheless, whereas the mesophilic EcDHFR, the thermophilic Geobacillus stearothermophilus DHFR (BsDHFR), and the cold-adapted Moritella profunda DHFR (MpDHFR) are monomeric enzymes that cannot catalyze hydride transfer
Scheme 1. Schematic Description of Chemical Reaction Catalyzed by DHFR

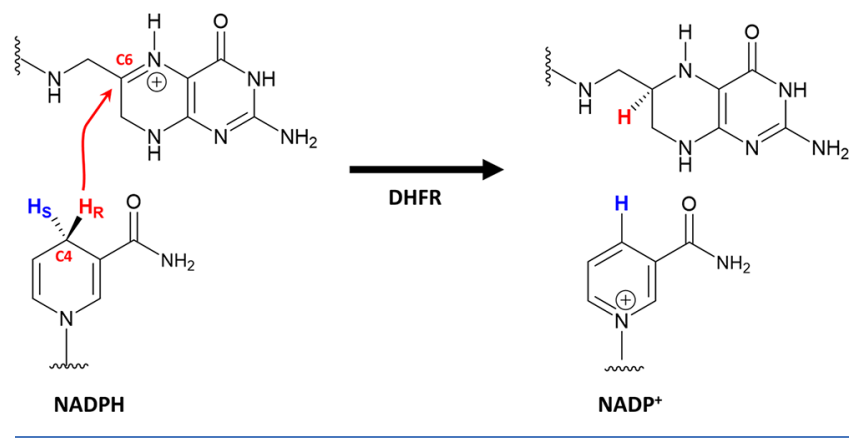

at temperatures above $65{ }^{\circ} \mathrm{C}$, the dimeric DHFR from the hyperthermophile Thermatoga maritima (TmDHFR) is active over a wide temperature range from 278 to well above $338 \mathrm{~K}$. The active form of TmDHFR is formed by two equivalent monomers with independent active sites and its thermal stability (melting temperature is $\sim 356 \mathrm{~K}$ ) is attributed to structural aspects related to surface loops involved in the

Received: March 26, 2019

Revised: May 10, 2019

Published: May 13, 2019 
contact area between the monomers (see Figure 1). ${ }^{6-8}$ The catalytic activity of TmDHFR is relatively low and the turnover

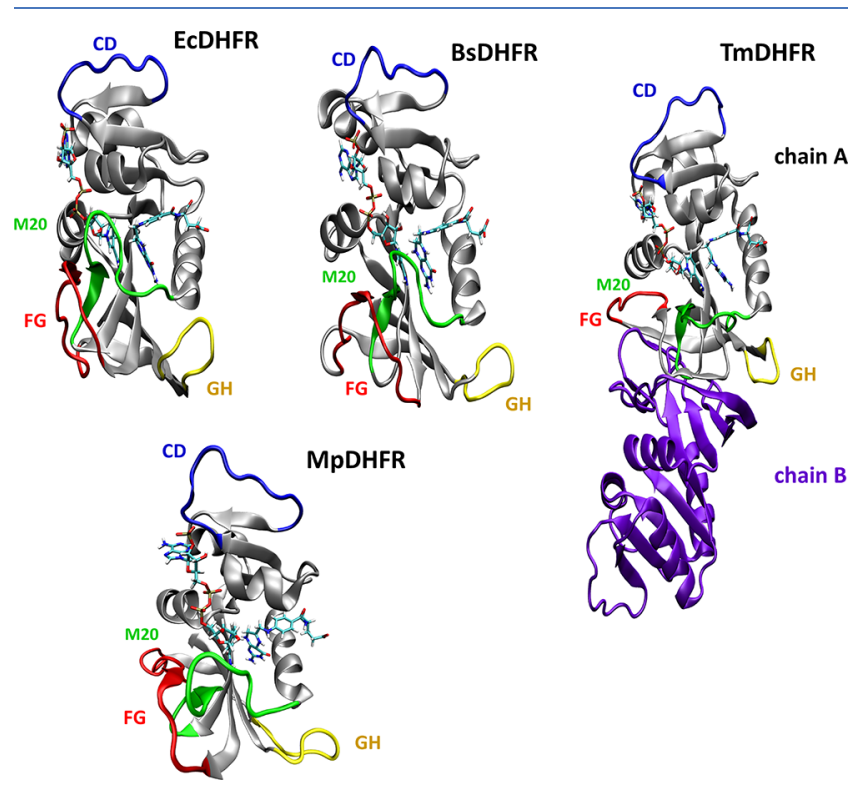

Figure 1. Cartoon representation of EcDHFR, BsDHFR, TmDHFR, and MpDHFR (PDB codes 1RX2, 1ZDR, 2ZZA, and 1D1G, respectively). In all four enzymes, folate and the cofactor NADPH are shown in ball and stick representations. In all DHFRs, the M20, FG, GH, and CD loops are highlighted in yellow, red, green, and blue, respectively. The M20 loop is in the occluded conformation in EcDHFR, BsDHFR, and MpDHFR but in the open conformation in dimeric TmDHFR, which is the only conformation that has been observed experimentally for TmDHFR. ${ }^{17,18}$

number $k_{\text {cat }}$ is $10-100$-fold lower than that of EcDHFR over the entire accessible temperature range. ${ }^{9}$ In monomeric DHFRs, there are several mobile segments on the protein surface such as the M20, FG and GH loops, which control the physical steps of the catalytic process such as substrate binding and product release. ${ }^{10}$ These loops are capable of switching between different conformations and this transition is especially important for the M20 loop due to its proximity to the active site (see Figure 1). ${ }^{11,12}$ In EcDHFR, the M20 loop is hydrogen bonded to residues in the FG loop and the two loops show coordinated motions during the catalytic cycle. In contrast, the FG and M20 loops of TmDHFR are part of the dimer interface ${ }^{13}$ rendering this enzyme less flexible than the monomeric counterparts. Although dimerization has been proposed as a strategy for enhancing enzymatic thermostability, its effect on catalysis has not been systematically explored for DHFRs. The lower catalytic power of enzymes with reduced flexibility such as TmDHFR has been proposed to support a possible link between protein dynamics and enzyme catalysis. ${ }^{2,3}$ However, Warshel and co-workers proposed that the lower catalytic efficiency is instead related to a larger reorganization energy needed to attain the transition state (TS) in the dimeric enzyme. ${ }^{14}$ Interestingly, recent analyses of some cold- and warm-adapted enzymes have shown that surface rigidification, as that observed in the dimer interface of TmDHFR, tunes the temperature dependence of enzymatic reaction rates by modifying the balance of the activation entropy and enthalpy, whereas the activation free energy at room temperature might remain unaltered. ${ }^{15,16}$
A combination of experimental and computational approaches has been used to clarify different aspects of biocatalysis including the relationship between catalysis and enzyme structure and flexibility. Kinetic isotope effects (KIEs) can be measured experimentally and predicted by computer simulations; they are one of the most efficient techniques to shed light onto the intimate aspects of enzymatic catalysis. ${ }^{19}$ The measurement of KIEs derived from the isotopic substitution of atoms directly involved in the chemical reaction (primary KIEs) can be used to propose and support reaction mechanisms at the molecular level. ${ }^{20-24}$ More recently, enzyme KIEs (EKIEs), resulting from the isotopic substitution of ${ }^{14} \mathrm{~N},{ }^{12} \mathrm{C}$, and nonexchangeable ${ }^{1} \mathrm{H}$ atoms of an enzyme with their heavier stable isotopes $\left({ }^{15} \mathrm{~N},{ }^{13} \mathrm{C}\right.$, and $\left.{ }^{2} \mathrm{H}\right)$ have been used to explore a possible link between the dynamics of the protein environment and the substrate during the chemical reaction $^{25-27}$ and thus can be useful to investigate the consequences of enzyme dimerization in catalysis. Isotopic substitution slows protein motions due to the mass increase, whereas within the Born-Oppenheimer approximation ${ }^{28}$ the electronic potential energy surface remains unperturbed. In general, heavy enzymes show a diminution in the rate constants of the chemical step relative to the light enzyme, ${ }^{6,29-31}$ rendering information about the influence of the protein and its dynamics in enzymatic catalysis. Experimental measurements on enzymatic KIEs have been carried out for several protein systems such as purine nucleoside phosphorylase, ${ }^{26} \mathrm{HIV}$ protease, ${ }^{32}$ alanine racemase, ${ }^{33}$ pentaerythritol tetranitrate reductase, ${ }^{34}$ and several dihydrofolate reductases (DHFRs). ${ }^{6,31,35-37}$ EcDHFR has been investigated extensively by theoretical and experimental measurmenets of KIEs, which have revealed the relationship between protein motions and catalysis. ${ }^{21,31,37-41}$ Combined in vitro and in silico work of enzyme KIEs has been performed for wild type EcDHFR ${ }^{31,35}$ and a catalytically compromised variant EcDHFR-N23PP/S148A. ${ }^{37}$ EKIEs were also measured for BsDHFR, ${ }^{36} \mathrm{MpDHFR},{ }^{42}$ and for Homo sapiens DHFR (hDHFR). ${ }^{43,44}$

Here, we report a theoretical investigation of the effect of protein motions on hydride transfer catalyzed by TmDHFR. The results of the simulations, carried out by means of hybrid $\mathrm{QM} / \mathrm{MM}$ methods in the framework of $\mathrm{TST}^{45,46}$ are compared with those obtained for other DHFRs $6,30,31,35,36,42$ and for its hypothetical monomeric version to elucidate the impact of protein dimerization and flexibility on the rate of hydride transfer. We have also computed enzymatic KIEs and compared our computational results with those obtained from previously published experimental work. ${ }^{6}$ The temperature dependence of the EKIEs and its possible correlation with the catalytic power of the different DHFRs and structural features such as protein flexibility are explored here in detail, shedding light on the relationship between protein dynamics, flexibility, and catalytic efficiency.

\section{METHODS}

2.1. Theoretical Framework. The calculation of the rate constant of the chemical step was based on transition state theory (TST) with the inclusion of tunneling contributions and recrossing effects as a preexponential factor. Thus, the equation to calculate the rate constant can be written as ${ }^{47-49}$ 


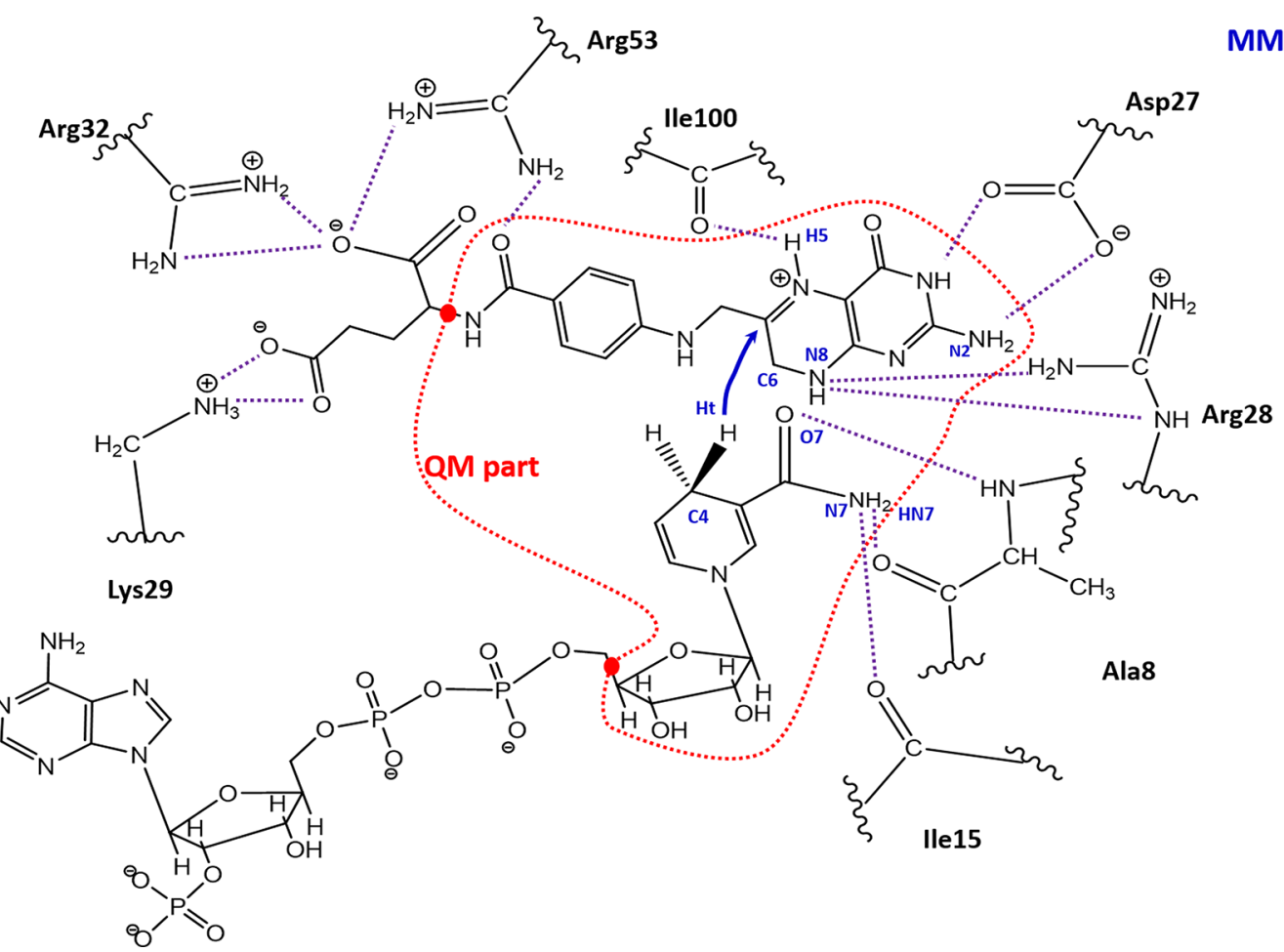

Figure 2. Schematic representation of the active site of TmDHFR and delineation of the QM/MM subsystem, where red points denote the position of the quantum link atoms.

$$
k_{\text {theor }}(T)=\Gamma(T, \xi) \frac{k_{\mathrm{B}} T}{h} e^{-\left(\frac{\Delta G_{\text {act }}^{\mathrm{eC}}(T, \xi)}{R T}\right)}=\frac{k_{\mathrm{B}} T}{h} e^{-\left(\frac{\Delta G_{\text {eff }}(T)}{R T}\right)}
$$

where $T$ is the temperature, $k_{\mathrm{B}}$ is the Boltzmann constant, $R$ is the ideal gas constant, $h$ is Planck's constant, $\Delta G_{\text {eff }}(T)$ corresponds to the effective activation free energy, which includes all the contributions to the rate constant and can be directly compared to the value derived from the experimentally measured rate constant. In eq $1, \Delta G_{\text {act }}^{\mathrm{QC}}$ represents the quasiclassical activation free energy obtained as a function of a selected reaction coordinate $\xi^{45}$

$$
\Delta G_{\text {act }}^{\mathrm{QC}}(T, \xi)=\Delta G_{\mathrm{act}}^{\mathrm{CM}}(T, \xi)+\Delta G_{\text {vib }}^{\mathrm{QM}}(T)
$$

where the $\Delta G_{\text {act }}^{\mathrm{CM}}(T, \xi)$ term is the activation free energy obtained from the classical potential of mean force (PMF) along the chosen reaction coordinate $(\xi)$ and $\Delta G_{\mathrm{vib}}^{\mathrm{QM}}(T)$ is a correction term due to the quantized nature of molecular vibrations (mainly zero-point energies). ${ }^{45,46,50}$ In our study, the reaction coordinate is associated to the hydride transfer from the donor atom (C4) of the cofactor $(\mathrm{NADPH})$ to the substrate's acceptor $\left(\mathrm{H}_{2} \mathrm{~F}\right)$ atom $(\mathrm{C} 6)$ and is defined as the antisymmetric combination between the breaking and forming bond distances (see Figure 2). Following the explanation of the terms of eq $1, \Gamma(T, \xi)$ corresponds to the temperaturedependent transmission coefficient, which can be expressed as

$$
G(T, \xi)=g(T, \xi) \cdot k(T)
$$

In eq $3, \gamma(T, \xi)$ corresponds to the classical recrossing transmission coefficient. This term is always lower than unity and takes into account the fact that some trajectories crossing the selected dividing surface (defined as $\xi=\xi^{\ddagger}$ ) from the reactants domain toward the products valley can recross back to the reactants valley. These recrossings are essentially due to the coupling of other degrees of freedom to the distinguished reaction coordinate $(\xi)^{51}$ and can be obtained by means of trajectories analysis or using Grote-Hynes theory. ${ }^{52} \kappa(T)$ corresponds to the tunneling coefficient that accounts for reactive trajectories that do not reach the classical threshold energy. ${ }^{45}$ Both terms of eq $3, \gamma(T, \xi)$ and $\kappa(T)$, are obtained from QM/MM simulations as detailed in the SI.

2.2. Simulation Details. The initial coordinates were taken from the X-ray crystal structure of T. maritima DHFR (PDB, 1D1G). ${ }^{12}$ TmDHFR is a dimeric protein with 164 residues in each of the two monomers. The protonation state of each residue was determined at $\mathrm{pH}=7$ using the PropKa 3.1 program for the whole dimer. ${ }^{53}$ Once the protonation state of each critical residue was determined, hydrogen atoms were added by means of the HBUILD tool included in the fDYNAMO package. ${ }^{54}$ Then, the system was placed inside a preequilibrated orthorhombic box of $100 \times 60 \times 60 \AA^{3}$ water molecules centered on the substrate-cofactor pair. Water molecules within $2.8 \AA$ of any non-hydrogen atom were removed. Five sodium cations were added in order to neutralize the total charge of the system and their initial position was selected according to the electrostatic potential created by the protein. The full system contained 35949 atoms, including the protein (5354 atoms in total), the substrate (protonated $\mathrm{H}_{2} \mathrm{~F}$ ) and cofactor $(\mathrm{NADH})$ in the active site of chain $\mathrm{A}$ and the cofactor in the active site of chain B (52 atoms of the substrate and $74 \times 2$ atoms of the cofactor, 200 atoms in total), 5 sodium ions, 154 crystal water molecules, and 9976 solvation water molecules (30390 atoms). Heavy TmDHFR was prepared in silico by modifying the masses of all ${ }^{14} \mathrm{~N},{ }^{12} \mathrm{C}$, and nonexchangeable ${ }^{1} \mathrm{H}$ atoms to those of ${ }^{15} \mathrm{~N},{ }^{13} \mathrm{C}$, and ${ }^{2} \mathrm{H}$, respectively. The ratio between the masses of the simulated heavy and light enzymes was 1.1143, which corresponds to the $100 \%$ isotope incorporation and 
Table 1. Contributions to the Rate Constant at 278, 298, 318, and $338 \mathrm{~K}$ Due to Recrossing $(\gamma)$, Tunneling ( $\kappa)$, Classical Mechanical Free Energy Barrier $\left(\Delta G_{\text {act }}^{\mathrm{CM}}\right)$, Quasi-Classical Free Energy of Activation $\left(\Delta G_{\text {act }}^{Q C}\right)$, and Effective Phenomenological Free Energies of Activation $\left(\Delta G_{\text {eff }}^{\ddagger}\right)^{a}$

\begin{tabular}{lcccccc} 
& $\gamma(T, \xi)$ & $\kappa(T)$ & $\Delta \mathrm{G}_{\text {act }}^{\mathrm{CM}}(\mathrm{T}, \xi)$ & $\Delta \mathrm{G}_{\text {act }}^{\mathrm{QC}}(T, \xi)$ & $\Delta G_{\text {eff }}^{\ddagger}(T)$ & \multicolumn{1}{c}{$\Delta G_{\text {exp }}^{\ddagger}$} \\
$\begin{array}{l}T=278 \mathrm{~K} \\
\text { light }\end{array}$ & $0.61 \pm 0.02$ & $5.5 \pm 0.8$ & $17.9 \pm 0.7$ & $16.02 \pm 0.75$ & $15.4 \pm 1.6$ & $17.89 \pm 0.01$ \\
heavy & $0.59 \pm 0.01$ & $5.1 \pm 0.8$ & & $16.05 \pm 0.74$ & $15.4 \pm 1.6$ & $17.89 \pm 0.01$ \\
$T=298 \mathrm{~K}$ & & & & & \\
light & $0.61 \pm 0.02$ & $4.5 \pm 0.6$ & $18.1 \pm 0.6$ & $16.25 \pm 0.65$ & $15.7 \pm 1.4$ & $18.44 \pm 0.01$ \\
heavy & $0.60 \pm 0.01$ & $4.2 \pm 0.6$ & & $16.27 \pm 0.64$ & $15.7 \pm 1.4$ & $18.45 \pm 0.01$ \\
$T=318 \mathrm{~K}$ & & & & & \\
light & $0.59 \pm 0.02$ & $3.8 \pm 0.4$ & $18.5 \pm 0.6$ & $16.68 \pm 0.65$ & $16.2 \pm 1.2$ & $18.85 \pm 0.01$ \\
heavy & $0.58 \pm 0.01$ & $3.7 \pm 0.5$ & & $16.70 \pm 0.64$ & $16.2 \pm 1.3$ & \\
$T=338 \mathrm{~K}$ & & & & & & \\
light & $0.57 \pm 0.02$ & $3.5 \pm 0.5$ & $18.7 \pm 0.4$ & $16.90 \pm 0.55$ & $16.4 \pm 1.3$ & $19.35 \pm 0.01$ \\
heavy & $0.56 \pm 0.02$ & $3.4 \pm 0.4$ & & $16.92 \pm 0.54$ & $16.5 \pm 1.2$ & $19.36 \pm 0.02$
\end{tabular}

${ }^{a}$ Values derived from the experimental rate constants ${ }^{6}$ are also provided $\left(\Delta G_{\text {exp }}^{\ddagger}\right)$. All free energies are given in kcal.mol ${ }^{-1}$. The errors bars given for theoretical predictions correspond to the standard deviation. The error bars for the experimental free energy correspond to the standard deviation obtained from the statistical error of the rate constant after applying propagation rule to TST equation.

close to the experimentally observed molecular weight increase $(98 \%)^{6}$

The whole system was divided into a QM part and a MM part (Figure 2) to perform combined QM/MM calculations. The quantum subsystem contained 76 atoms, including parts of the cofactor (nicotinamide ring and ribose) and the substrate (pteridine ring and the $N$-methylene-substituted $p$ aminobenzoyl, pABA) of chain A. Two hydrogen link atoms ${ }^{55}$ were used to saturate the valence at the $Q M / M M$ boundary. The quantum atoms were described by the AM1 Hamiltonian, ${ }^{56}$ modified using specific reaction parameters (denoted as AM1-SRP) developed previously for DHFR by Major and co-workers that provide excellent activation free energies and geometries. ${ }^{57}$ The protein atoms, the cofactor of the second active site, and the ions were described by the OPLS-AA ${ }^{58}$ force field, whereas water molecules were described using the TIP3P potential. ${ }^{59}$ Cutoffs for the nonbonding interactions were applied using a switching function within a range of 13.0 to $9.0 \AA$. Periodic boundary conditions were employed combined with the minimum image convention in all the simulations.

One-dimensional PMFs were computed using the antisymmetric combination of distances describing the hydride transfer, $\xi=d\left(\mathrm{C}_{4}-\mathrm{H}_{\mathrm{t}}\right)-d\left(\mathrm{H}_{\mathrm{t}}-\mathrm{C}_{6}\right)$, as the reaction coordinate. The umbrella sampling approach was used ${ }^{60}$ with the system restrained to remain close to the desired values of the reaction coordinate by means of the addition of a harmonic potential with a force constant of $2500 \mathrm{~kJ} \mathrm{~mol}^{-1} \mathrm{~A}^{-2}$, which allows good overlap between windows. The reaction coordinate was explored in a range from -2.1 to $1.75 \AA$ with a total number of windows of 55. The probability distributions obtained from $\mathrm{MD}$ simulations within each individual window were combined by means of the weighted histogram analysis method (WHAM). ${ }^{61}$ For each simulation window, $100 \mathrm{ps}$ of relaxation and $100 \mathrm{ps}$ of production were run with a time step of $0.5 \mathrm{fs}$ in the canonical ensemble (NVT) using the Langevin-Verlet integrator. This procedure was repeated for 11 temperatures ranging from 278 to $338 \mathrm{~K}$. Three PMFs were computed at the AM1-SRP/MM level for each temperature to obtain well-converged averaged classical mechanical activation free energy barriers. The classical mechanical activation free energies presented in Table 1 were obtained as the average of three different PMFs for each of the simulated temperatures $(278,298,318$ and $338 \mathrm{~K}$ ) (Figure S1). For other temperatures, the classical mechanical activation free energy was obtained from just one PMF.

\section{RESULTS}

3.1. Activation Free Energies. QM/MM simulations were run for the light and heavy versions of TmDHFR. All the terms in eqs 1-3 (see Methods), obtained at 278, 298, 318, and $338 \mathrm{~K}$, are listed in Table 1 . Results obtained at other temperatures $(283,288,293,303,308,318$, and $328 \mathrm{~K})$ are provided in the Supporting Information (see Tables S2 and S3).

Our theoretical predictions of the effective free energy barriers for hydride transfer in TmDHFR underestimate the free energy barrier by $\sim 2 \mathrm{kcal} \cdot \mathrm{mol}^{-1}$ calculated from the experimentally determined rate constants (Table 1) but reproduce the observed increase of the barrier with temperature. A linear fit of the calculated activation free energies versus temperature (Figure S2) provides an activation entropy in good agreement with the value derived from the experimental rate constants, ${ }^{6}-18.8 \pm 1$ versus $-22.6 \pm 0.6$ $\mathrm{cal} \cdot \mathrm{mol}^{-1} \cdot \mathrm{K}^{-1}$, respectively. It is interesting to note that the free energy barrier increases less with temperature in TmDFHFR than in EcDHFR for which an activation entropy of $-26.1 \pm$ $0.6 \mathrm{cal} \cdot \mathrm{mol}^{-1} \cdot \mathrm{K}^{-1}$ was reported. ${ }^{62}$ This is related to the fact that TmDHFR is a warm-adapted enzyme that generally shows larger activation enthalpies and less negative activation entropies than its cold-adapted homologues. ${ }^{15,16}$ Our calculations also agree with the experimental observation that at room temperature TmDHFR catalyzes the hydride transfer less efficiently than EcDHFR. ${ }^{17}$ Using the same theoretical approach, we estimated that the effective activation free energy barrier in EcDHFR at $298 \mathrm{~K}$ is $14.3 \mathrm{kcal} \cdot \mathrm{mol}^{-1}$, 31 about 1.5 $\mathrm{kcal} \cdot \mathrm{mol}^{-1}$ smaller than that for the TmDHFR catalyzed reaction.

Dimerization was initially proposed as the reason for the reduced catalytic efficiency of TmDHFR. ${ }^{17}$ However, kinetic measurements on monomeric and dimeric forms of a TmDHFR variant in which Val11 was replaced by Asp found that both forms presented a similar rate for hydride transfer. ${ }^{41}$ It was then concluded that the lower rates of the reaction 
Table 2. Contributions to the Rate Constant of Hydride Transfer in the Monomer of TmDHFR at 298 K Due to Recrossing $(\gamma)$, Tunneling $(\kappa)$, Classical Mechanical Free Energy Barrier $\left(\Delta G_{\text {act }}^{\mathrm{CM}}\right)$, Quasi-Classical Free Energy of Activation $\left(\Delta G_{\text {act }}^{\mathrm{QC}}\right)$, and Effective Phenomenological Free Energies of Activation $\left(\Delta G_{\mathrm{eff}}^{\ddagger}\right)^{a}$

$\begin{array}{cccccc} & \gamma(T, \xi) & \kappa(T) & \Delta G_{\text {act }}^{\mathrm{CM}}(T, \xi) & \Delta G_{\text {act }}^{\mathrm{eC}}(T) & \Delta G_{\text {eff }}^{\ddagger}(T) \\ \text { light } & 0.55 \pm 0.02 & 4.2 \pm 0.3 & 18.9 \pm 0.6 & 17.04 \pm 0.7 & 16.5 \pm 1.2 \\ \text { heavy } & 0.54 \pm 0.01 & 4.1 \pm 0.5 & & 17.08 \pm 0.7 & 16.6 \pm 1.2 \\ { }^{a} \text { All free energies are given in } \mathrm{kcal} \cdot \mathrm{mol}^{-1} \text {. } & & & & \end{array}$

catalyzed by wild type dimeric TmDHFR relative to that of EcDHFR are not a direct consequence of dimerization but stem from the modifications that allow dimerization. ${ }^{63}$ In order to shed light on this debate, QM/MM simulations on the hypothetical TmDHFR monomer were performed using the same theoretical approach as was used for the dimer. The results at $298 \mathrm{~K}$ are presented in Table 2 (see Table S4 for results also at 278 and $318 \mathrm{~K}$ ) and show that the values for the free energy barriers of the monomeric and dimeric forms are identical with the difference of about $0.8 \mathrm{kcal} \cdot \mathrm{mol}^{-1}$ within the statistical uncertainties of the methodology. As mentioned before, previous theoretical studies predicted significantly smaller rate constants for the monomeric form ${ }^{18}$ which is in disagreement with later experimental observations. ${ }^{41}$ These previous calculations also predicted an increase in the tunneling transmission coefficient in the monomer of TmDHFR relative to the dimer, an effect that is not reproduced in our calculations. Our results confirm that dimerization itself does not alter the catalytic efficiency at room temperature or the contribution of tunneling. Instead, dimerization rigidifies some structural elements of the protein (the loops involved in the dimer interface, as discussed below), which keeps the structural integrity of the protein at higher temperatures and shifts the activation enthalpy-entropy balance to a less negative activation entropy and a larger activation enthalpy. A linear fit of the activation free energies obtained for the monomer versus temperature (Figure S3) revealed an activation entropy of $-50 \pm 3 \mathrm{cal} \cdot \mathrm{mol}^{-1} \cdot \mathrm{K}^{-1}$, clearly larger in absolute value than for the dimer. Although the number of points used in the fit is small, the observation of an increased value for the activation entropy in the monomer with respect to the dimer is also supported by other observations (see below). This effect of dimerization on the activation entropy-enthalpy balance results in an activation free energy for the dimeric TmDHFR that increases less steeply with temperature, providing in this way larger catalytic rate constants at higher temperatures. This is clearly a beneficial effect of dimerization for thermophilic enzymes.

3.2. Structural Analysis. The geometries of the RS and TS in TmDHFR at different simulated temperatures are provided in Table S5. In general, the description of the chemical reaction is very similar in TmDHFR and EcDHFR (see Table S6 for averaged geometrical values of EcDHFR), but the hydride donor-acceptor distance at the RS is slightly larger for TmDHFR than for EcDHFR with $4.2 \pm 0.1$ and 3.9 $\pm 0.2 \AA$, respectively. This feature, also observed by Warshel and co-workers, ${ }^{14}$ can be explained because the open conformation observed for the M20 loop in TmDHFR allows more water molecules to enter the active site. This is illustrated in Figure 3 that shows the presence of water molecules during MD simulations of TmDHFr and EcDHFR and can be quantified by the radial distribution function around the N5 atom of the substrate (Figure S4). Water molecules stabilize the RS better than the TS because the charge on the substrate
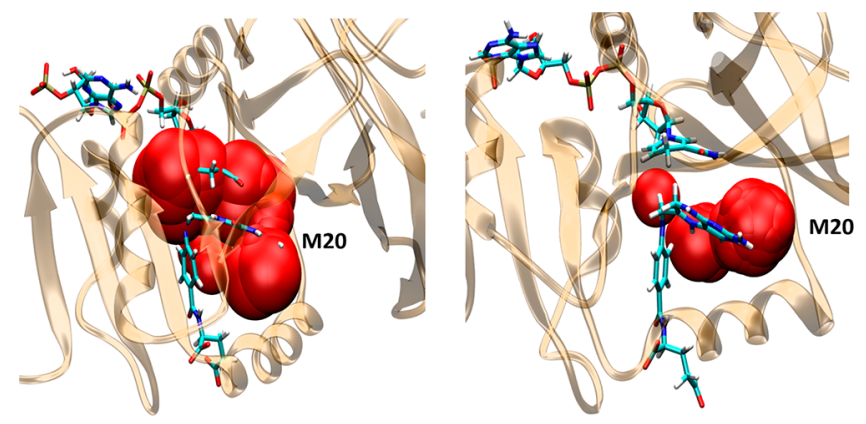

Figure 3. Representative snapshots of the RS of the hydride transfer catalyzed by TmDHFR (left side) and EcDHFR (right side). ${ }^{37}$ Locations of water molecules found during simulations close to the N5 atom of the substrate are represented by space filling red balls.

is more localized. This is reflected in the position of the first peak of the radial distribution function that is shifted from 5.1 to $5.4 \AA$ when passing from RS to TS in TmDHFR. In general, water molecules present in active sites can follow the electronic changes in the chemical system but at the cost of a larger reorganization energy, which is then translated into a larger activation free energy and a lower reaction rate for the chemical reaction. ${ }^{14}$ This has also been expressed as the importance of "not being in water for catalysis". ${ }^{64}$ Major and co-workers found that the sequestering of water molecules to the active site of DHFRs is correlated with a larger catalytic efficiency. ${ }^{65}$ The less negative activation entropy found in TmDHFR can also be partly due to the presence of water molecules in the active site, because these water molecules become more disordered in TS relative to RS (as demonstrated by the aforementioned shift in the radial distribution function).

In EcDHFR, the M20 loop is in a closed conformation during the hydride transfer step. ${ }^{10,66}$ In our previous combined theoretical and experimental EcDHFR work, it was shown how some interactions of residues belonging to this loop, such as Ala19 and Met20, preferentially stabilize the TS (for key interatomic distances in EcDHFR see Table S6). ${ }^{37}$ Impairing these interactions at the TS, as observed in EcDHFR-N23PP/ S148A, reduces the rate constant by more than an order of magnitude. ${ }^{37}$ In TmDHFR, the M20 loop appears to be fixed in an open conformation favored not only by intersubunit but also by intraloop interactions, such as the hydrogen bond between Ser17 and Trp22 that does not exist in EcDHFR (there is no a hydrogen bond donor at position 17 in the E. coli enzyme). The closed conformation prevents the formation of some interactions between the residues of the M20 loop and the substrate or the cofactor that could stabilize the TS, as deduced from the comparison of the intermolecular distances reported in Tables S5 and S6. As a reference, residue Met20 in EcDHFR approaches the cofactor to about $2.0 \pm 0.5 \AA$ when passing from RS to TS, while none of the residues in TmDHFR see such a displacement. This contributes to the 

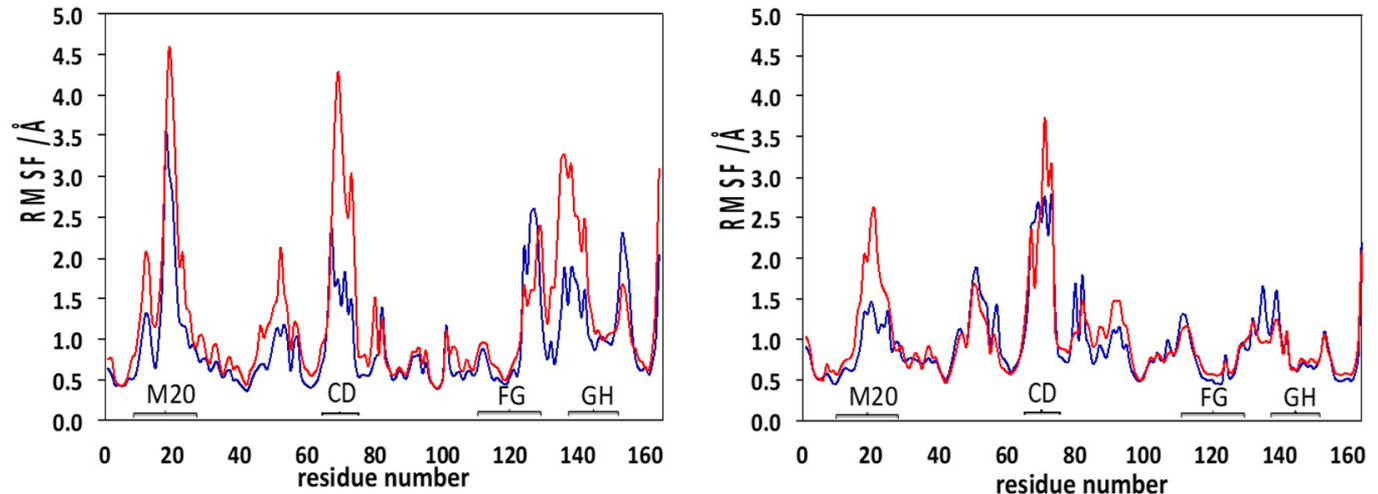

Figure 4. RMSF (in $\AA$ ) of the $\mathrm{C}^{\alpha}$ atoms obtained after $50 \mathrm{~ns}$ of classical MD simulation at the Michaelis complex of the hypothetical monomer (left) and the dimer (right) of TmDHFR at two temperatures: $298 \mathrm{~K}$ (blue lines) and $338 \mathrm{~K}$ (red lines).
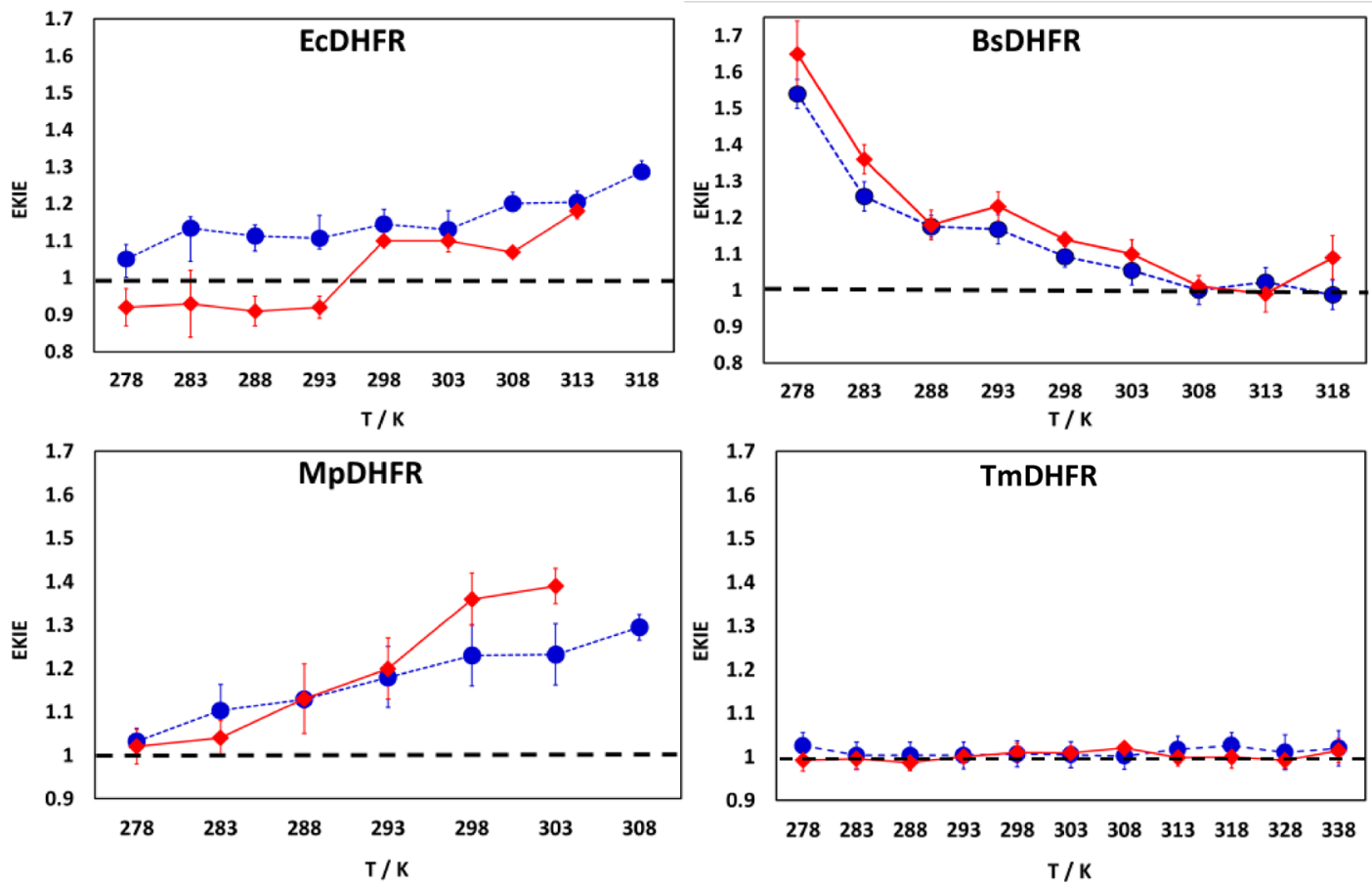

Figure 5. Temperature dependence of experimental (solid red lines) and calculated (dashed blue lines) EKIEs for EcDHFR, BsDHR, MpDHFR, and TmDHFR. Errors bars are shown as vertical lines.

reduction in the catalytic efficiency of the enzyme by increasing the activation enthalpy due to the lack of some favorable interactions. The enthalpy increase is partly compensated by the fact that as less interactions are established between the substrate and the enzyme the protein is less stiffened when passing from RS to TS, contributing to a less negative activation entropy, as determined in the previous section.

Although dimerization itself does not significantly alter the rate of hydride transfer at room temperature, it is nevertheless important for the thermal stability of TmDHFR and thus indirectly maintains its catalytic activity at higher temperatures. Both experimental measurements ${ }^{6}$ and the simulations presented here (Table 1 ) reveal that the activation free energy of TmDHFR increases less steeply with temperature than for other dihydrofolate reductase catalyzed reactions. ${ }^{62}$ The values defining the reaction coordinate, the donor-acceptor distance and the distances for the protein interactions with the substrate and the cofactor are kept quite constant over the temperature range investigated here (Table S5). In other words, the active site of TmDHFR is preorganized for catalysis, albeit less efficiently, than in other DHFRs such as EcDHFR. This preorganization of TmDHFR is thermally stable, which appears to be a distinctive feature of this enzyme within the family of DHFRs.

To investigate the impact of dimerization on the thermal stability of the protein structure, we computed the root mean squared fluctuations (RMSFs) of the $\mathrm{C}^{\alpha}$ atoms of the monomeric and dimeric versions of TmDHFR at 298 and $338 \mathrm{~K}$ (Figure 4). When the temperature increases from 298 to $338 \mathrm{~K}$, the monomer shows a clear increase in the value of the RMSFs, in particular for the M20, GH, and CD loops. An increase of the temperature is translated into larger fluctuations of these loops that could eventually lead to denaturation of the monomeric form at larger temperatures. This increased flexibility with the temperature is dramatically attenuated in the dimer. Thus, our simulations suggest that dimerization makes the whole protein structure more rigid, not only the active site, and is thus more resistant to melting which is in 
agreement with the experimental results. In this way, the catalytic function of TmDHFR can be preserved at higher temperatures, which is not the case of EcDHFR or the hypothetical monomeric form of TmDHFR.

Analysis of cross-correlation motions between residues for the dimeric and monomeric forms of TmDHFR (see Figure S5) reveals a subtle mechanism for the coupling between the two monomers in the dimer. Dimerization introduces new correlations between motifs of the different subunits. In particular, strong positive correlations are observed between loops FG and GH belonging to different subunits. This is not unexpected because these loops are located in the dimerization area. Interestingly, the motions of these loops are correlated with the catalytically important M20 loops. The M20 loops of the two subunits also display positive cross-correlations between them. The coupling observed between the motions of the loops of both subunits could be at the origin of the thermal stability of the dimer observed in Figure 4. In particular, analysis of the backbone dihedral angles of the M20 loop shows that this loop can adopt different conformations in the monomeric and dimeric forms of TmDHFR (see Figure S6).

3.3. Enzyme Kinetic Isotope Effects. Enzyme KIEs and their temperature dependence can usually be well reproduced from the ratio of the corresponding transmission coefficients computed for the light and heavy enzymes $\left(\gamma^{\mathrm{LE}} / \gamma^{\mathrm{HE}}\right)$. This approach has previously been employed to analyze with good accuracy the changes in reactivity upon mass alteration of wild type EcDHFR, ${ }^{35}$ EcDHFR-N23PP-S148A, ${ }^{37}$ BsDHFR, $^{36}$ and MpDHFR. $^{42}$ As can be seen in Table 1 , the transmission coefficients are the only parameters appearing in the TST rate equation that are significantly different between the light and heavy versions of the enzyme when the statistical uncertainties are considered. Within our approach, the reaction coordinate depends exclusively on the degrees of freedom of the chemical system (the substrate and the cofactor) and hence any nonequilibrium effects of protein dynamics should be captured in this transmission coefficient.

Figure 5 shows the temperature dependence of EKIEs experimentally determined for EcDHFR, BsDHFR, MpDHFR, and TmDHFR (dimer) together with our theoretical predictions for all of these four enzymes. In particular, experimental and theoretical values for EcDHFR, BsDHFR, and MpDHFR were taken from refs 31, 36, and 42, experimental values for dimeric TmDHFR were taken from ref 6, whereas the theoretical values of TmDHFR were obtained from the data shown in Tables 1 and S2.

The temperature dependence of both experimental and theoretical EKIEs shows that they are close to unity at the optimum working temperature of each DHFR enzyme (see Figure 5). EKIEs of EcDHFR (a mesophilic enzyme) approach unity at room temperature; for BsDHFR (a thermophilic enzyme), this value is reached at higher temperatures, whereas for MpDHFR (an enzyme from a cold-adapted organism) the EKIEs approach 1 at low temperature. All enzymes have been optimized by evolution to work at their physiological temperatures. This means that their three-dimensional structure is preorganized to catalyze the chemical reaction, requiring a minimal amount of reorganization, that is, motions in the enzymatic structure, to reach the TS at the temperature where the host organism thrives. If the participation of protein motions is minimized, the change from a light version of an enzyme to the heavy counterpart has minimal effects on the reaction rate constants and the EKIEs are close to unity. If the working temperature is changed, then different conformational states can become accessible to the enzyme. These conformations will not have been optimized at nonphysiological temperatures, so that increased dynamics is required to reach the TS, which in turn will increase the impact of the protein mass on the reaction rate constant.

The only exception for this general behavior is TmDHFR for which EKIEs are close to unity over the whole range of temperatures studied here. In this regard, it is interesting to compare the different behaviors of two thermophilic enzymes, namely BsDHFR (a moderately thermophilic monomeric enzyme) and TmDHFR (a hyperthermophilic dimeric enzyme) (Figure 5). BsDHFR and TmDHFR follow different strategies in the adaptation to high temperatures. Thermal adaptation in BsDHFR is achieved by removing themolabile residues and extending secondary structural elements, which provokes some elements to become significantly more flexible than in the mesophilic EcDHFR. ${ }^{36}$ The structure of TmDHFR on the other hand is less sensitive to an increase in temperature (Figure 4). The most flexible motifs such as the loops are locked in particular conformations at the dimer interface and the structural integrity of the enzyme is preserved. As a result, the participation of protein motions is minimized over the whole range of temperatures studied and the EKIEs are always close to unity. Another factor that contributes to the invariance of the EKIEs in TmDHFR is the increased number of water molecules in the active site. These are responsible of the environmental reorganization taking place during the chemical step and are not affected by the change of the mass of the protein. Their contribution to the reorganization of the active site during hydride transfer remains unaltered when going from light to the heavy TmDHFR. Together rigidity of the TmDHFR structure and the presence of water molecules in the active site explain the observed invariance of EKIEs with temperature. Interestingly, an in silico estimation of the solvent $\mathrm{KIE}$ as the ratio between the transmission coefficient obtained in deuterated and normal water, gives a value of $1.13 \pm 0.03$, reflecting the impact of the dynamics of active site water molecules in the chemical step.

\section{CONCLUSIONS}

Theoretical simulations at different temperatures have been used to rationalize the behavior of TmDHFR as compared to the other variants of the DHFR family and a hypothetical monomeric form of TmDHFR that has never been observed experimentally.

The results presented here show that dimerization is not responsible for the reduced catalytic efficiency of TmDHFR; the reduced efficiency is likely to be a consequence of changes introduced in the primary sequence of TmDHFR that stabilize the dimer. The M20 loop is central to rationalize the differences between TmDHFR and other members of the DHFRS family. In TmDHFR, it is in the open form preventing the formation of some stabilizing interactions in the TS found in EcDHFR. In addition, the open conformation allows more water molecules to enter the active site of TmDHFR. These water molecules stabilize RS relative to TS and this is reflected in a larger change in the hydride donor-acceptor distance when going from the RS to the TS. This effect contributes to the increase in the free energy barrier of the reaction. The presence of water molecules also helps diminish the absolute 
value of the activation entropy, a property of TmDHFR that makes it more catalytically competent at high temperatures.

Dimerization confers on TmDHFR the ability to remain catalytically active at high temperatures. The RMSFs of TmDHFR show a reduced flexibility of the dimeric form compared to the monomer. This reduction is more evident at higher temperatures, where thermal fluctuations can lead to unfolding of the enzyme. However, some protein flexibility is a prerequisite for the reaction so that the active site can accommodate the charge redistribution on the path to the TS. ${ }^{37}$ The absence of flexibility in TmDHFR can be partially compensated by an active site that seems to be almost completely preorganized in the Michaelis complex. No significant changes are observed when comparing proteinsubstrate distances at RS and TS at different temperatures; this means that the pattern of protein interactions with the chemical system, despite not being as optimal as in other DHFRs, are already present in the Michaelis complex.

This picture of the role of protein flexibility in catalysis in TmDHFR is corroborated by the analysis of EKIEs. Analysis of the temperature dependence of both experimental and theoretical EKIEs obtained for other DHFRs shows that a value close to unity is reached at their respective physiological temperatures; the values of the enzyme KIEs increase when moving toward nonphysiological temperatures. In the hyperthermophilic TmDHFR on the other hand, EKIEs remain close to unity over the whole temperature range, indicating a minor participation of protein motions during barrier crossing.

\section{ASSOCIATED CONTENT}

\section{S Supporting Information}

The Supporting Information is available free of charge on the ACS Publications website at DOI: 10.1021/acscatal.9b01250.

Methodological details for the calculation of tunneling and recrossing contributions; tables with the temperature dependence of the contributions to the rate constant for dimeric and monomeric TmDHFR; averaged geometries for reactants and transition states in TmDHFR and EcDHFR; figure of the temperature dependence of the activation free energy in TmDHFR and the radial distribution functions around the N5 atom in monomeric and dimeric TmDHFR and in EcDHFR; representation of cross-correlation matrixes for monomeric and dimeric TmDHFR; Ramachandran plots for M20, FG, and GH loops in monomeric and dimeric TmDHFR and time evolution of backbone dihedral angles for residue 16 (PDF)

\section{AUTHOR INFORMATION}

\section{Corresponding Authors}

*E-mail: (I.T.) ignacio.tunon@uv.es.

*E-mail: (V.M.) moliner@uji.es.

*E-mail: (R.K.A.) AllemannRK@cardiff.ac.uk.

\section{ORCID $\odot$}

Iñaki Tuñón: 0000-0002-6995-1838

Vicent Moliner: 0000-0002-3665-3391

\section{Notes}

The authors declare no competing financial interest.

\section{ACKNOWLEDGMENTS}

This work was supported by the Spanish Ministerio de Economia y Competitividad and FEDER funds (projects PGC2018-094852-B-C2, CTQ2015-66223-C2, and CTQ2015-74523-JIN(AEI/FEDER)), Universitat Jaume I (project UJI-B2017-31), Generalitat Valenciana (AICO/ 2018/238), the U.S.A. National Institute of Health (Ref. No. NIH R01 GM065368) and the U.K.'s Biotechnology and Biological Sciences Research Council (BBSRC) (Grants BB/ J005266/1 and BB/L020394/1 (R.K.A.) The authors acknowledge computational resources from the Servei d'Informàtica of Universitat de Valencia.

\section{REFERENCES}

(1) Huang, P.-S.; Boyken, S. E.; Baker, D. The Coming of Age of de Novo Protein Design. Nature 2016, 537, 320-327.

(2) Kohen, A.; Cannio, R.; Bartolucci, S.; Klinman, J. P.; Klinman, J. P. Enzyme Dynamics and Hydrogen Tunnelling in a Thermophilic Alcohol Dehydrogenase. Nature 1999, 399, 496-499.

(3) Kohen, A.; Klinman, J. P. Protein Flexibility Correlates with Degree of Hydrogen Tunneling in Thermophilic and Mesophilic Alcohol Dehydrogenases. J. Am. Chem. Soc. 2000, 122, 10738-10739.

(4) Berman, M. H.; Frazer, A. C. Importance of Tetrahydrofolate and Atp in the Anaerobic O-Demethylation Reaction for Phenylmethylethers. Appl. Environ. Microb. 1992, 58, 925-931.

(5) Henzler-Wildman, K. A.; Lei, M.; Thai, V.; Kerns, S. J.; Karplus, M.; Kern, D. A hierarchy of Timescales in Protein Dynamics is Linked to Enzyme Catalysis. Nature 2007, 450, 913-916.

(6) Luk, L. Y.; Loveridge, E. J.; Allemann, R. K. Different Dynamical Effects in Mesophilic and Hyperthermophilic Dihydrofolate Reductases. J. Am. Chem. Soc. 2014, 136, 6862-6865.

(7) Loveridge, E. J.; Allemann, R. K. The Temperature Dependence of the Kinetic Isotope Effects of Dihydrofolate Reductase from Thermotoga Maritima is Influenced by Intersubunit Interactions. Biochemistry 2010, 49, 5390-5396.

(8) Guo, J.; Loveridge, E. J.; Luk, L. Y.; Allemann, R. K. Effect of Dimerization on Dihydrofolate Reductase Catalysis. Biochemistry 2013, 52, 3881-3887.

(9) Maglia, G.; Javed, M. H.; Allemann, R. K. Hydride Transfer During Catalysis by Dihydrofolate Reductase from Thermotoga Maritima. Biochem. J. 2003, 374, 529-535.

(10) Sawaya, M. R.; Kraut, J. Loop and Subdomain Movements in the Mechanism of Escherichia Coli Dihydrofolate Reductase: Crystallographic Evidence. Biochemistry 1997, 36, 586-603.

(11) Dams, T.; Auerbach, G.; Bader, G.; Jacob, U.; Ploom, T.; Huber, R.; Jaenicke, R. The Crystal Structure of Dihydrofolate Reductase from Thermotoga Maritima: Molecular Features of Thermostability. J. Mol. Biol. 2000, 297, 659-672.

(12) Behiry, E. M.; Luk, L. Y. P.; Matthews, S. M.; Loveridge, E. J.; Allemann, R. K. Role of the Occluded Conformation in Bacterial Dihydrofolate Reductases. Biochemistry 2014, 53, 4761-4768.

(13) Dams, T.; Bohm, G.; Auerbach, G.; Bader, G.; Schurig, H.; Jaenicke, R. Homo-dimeric Recombinant Dihydrofolate Reductase from Thermotoga Maritima Shows Extreme Intrinsic Stability. Biol. Chem. 1998, 379, 367-371.

(14) Roca, M.; Liu, H.; Messer, B.; Warshel, A. On the Relationship between Thermal Stability and Catalytic Power of Enzymes. Biochemistry 2007, 46, 15076-15088.

(15) Isaksen, G. V.; Åqvist, J.; Brandsdal, B. O. Enzyme Surface Rigidity Tunes the Temperature Dependence of Catalytic Rates. Proc. Natl. Acad. Sci. U. S. A. 2016, 113, 7822-7827.

(16) Åqvist, J.; Isaksen, G. V.; Brandsdal, B. O. Computation of Enzyme Cold Adaptation. Nat. Rev. Chem. 2017, 1, 0051.

(17) Maglia, G.; Javed, M. H.; Allemann, R. K. Hydride Transfer During Catalysis by Dihydrofolate Reductase from Thermotoga Maritima. Biochem. J. 2003, 374, 529-535. 
(18) Pang, J. Y.; Pu, J. Z.; Gao, J. L.; Truhlar, D. G.; Allemann, R. K. Hydride Transfer Reaction Catalyzed by Hyperthermophilic Dihydrofolate Reductase is Dominated by Quantum Mechanical Tunneling and is Promoted by Both Inter- and Intramonomeric Correlated Motions. J. Am. Chem. Soc. 2006, 128, 8015-8023.

(19) Kohen, A. Kinetic Isotope Effects as Probes for Hydrogen Tunneling, Coupled Motion and Dynamics Contributions to Enzyme Catalysis. Prog. React. Kinet. Mech. 2003, 28, 119-156.

(20) Seravalli, J.; Huskey, W. P.; Schowen, K. B.; Schowen, R. L. Catalytic and Regulatory Strategies of Thermophilic LactateDehydrogenase - Microscopic Rate Constants from Kinetic Isotope Effects. Pure Appl. Chem. 1994, 66, 695-702.

(21) Schramm, V. L. Enzymatic Transition State Poise and Transition State Analogues. Acc. Chem. Res. 2003, 36, 588-596.

(22) García-Viloca, M.; Truhlar, D. G.; Gao, J. Reaction-Path Energetics and Kinetics of the Hydride Transfer Reaction Catalyzed by Dihydrofolate Reductase. Biochemistry 2003, 42, 13558-13575.

(23) Cleland, W. W. The Use of Isotope Effects to Determine Enzyme Mechanisms. J. Biol. Chem. 2003, 278, 51975-51984.

(24) Park, H.; Girdaukas, G. G.; Northrop, D. B. Effect of Pressure on a Heavy-Atom Isotope Effect of Yeast Alcohol Dehydrogenase. J. Am. Chem. Soc. 2006, 128, 1868-1872.

(25) Silva, R. G.; Murkin, A. S.; Schramm, V. L. Femtosecond Dynamics Coupled to Chemical Barrier Crossing in a BornOppenheimer Enzyme. Proc. Natl. Acad. Sci. U. S. A. 2011, 108, $18661-18665$.

(26) Kipp, D. R.; Silva, R. G.; Schramm, V. L. Mass-Dependent Bond Vibrational Dynamics Influence Catalysis by HIV-1 Protease. J. Am. Chem. Soc. 2011, 133, 19358-19361.

(27) Swiderek, K.; Ruiz-Pernía, J. J.; Moliner, V.; Tuñón, I. Heavy Enzymes - Experimental and Computational Insights in Enzyme Dynamics. Curr. Opin. Chem. Biol. 2014, 21, 11-18.

(28) Born, M.; Oppenheimer, R. Zur Quantentheorie der Molekeln. Ann. Phys. 1927, 389, 457-484.

(29) Luk, L. Y.; Loveridge, E. J.; Allemann, R. K. Protein Motions and Dynamic Effects in Enzyme Catalysis. Phys. Chem. Chem. Phys. 2015, 17, 30817-30827.

(30) Behiry, E. M.; Evans, R. M.; Guo, J.; Loveridge, E. J.; Allemann, R. K. Loop Interactions During Catalysis by Dihydrofolate Reductase from Moritella Profunda. Biochemistry 2014, 53, 4769-4774.

(31) Luk, L. Y. P.; Ruiz-Pernía, J. J.; Dawson, W. M.; Roca, M.; Loveridge, E. J.; Glowacki, D. R.; Harvey, J. N.; Mulholland, A. J.; Tuñón, I.; Moliner, V.; Allemann, R. K. Unraveling the Role of Protein Dynamics in Dihydrofolate Reductase Catalysis. Proc. Natl. Acad. Sci. U. S. A. 2013, 110, 16344-16349.

(32) Kipp, D. R.; Silva, R. G.; Schramm, V. L. Mass-Dependent Bond Vibrational Dynamics Influence Catalysis by HIV-1 Protease. J. Am. Chem. Soc. 2011, 133, 19358-19361.

(33) Toney, M. D.; Castro, J. N.; Addington, T. A. Heavy-Enzyme Kinetic Isotope Effects on Proton Transfer in Alanine Racemase. J. Am. Chem. Soc. 2013, 135, 2509-2511.

(34) Pudney, C. R.; Guerriero, A.; Baxter, N. J.; Johannissen, L. O.; Waltho, J. P.; Hay, S.; Scrutton, N. S. Fast Protein Motions are Coupled to Enzyme H-Transfer Reactions. J. Am. Chem. Soc. 2013, 135, 2512-2517.

(35) Ruiz-Pernía, J. J.; Luk, L. Y.; García-Meseguer, R.; Marti, S.; Loveridge, E. J.; Tuñón, I.; Moliner, V.; Allemann, R. K. Increased Dynamic Effects in a Catalytically Compromised Variant of Escherichia Coli Dihydrofolate Reductase. J. Am. Chem. Soc. 2013, 135, 18689-18696.

(36) Luk, L. Y.; Ruiz-Pernía, J. J.; Dawson, W. M.; Loveridge, E. J.; Tunón, I.; Moliner, V.; Allemann, R. K. Protein Isotope Effects in Dihydrofolate Reductase from Geobacillus Stearothermophilus Show Entropic-Enthalpic Compensatory Effects on the Rate Constant. J. Am. Chem. Soc. 2014, 136, 17317-17323.

(37) Luk, L. Y.; Ruiz-Pernía, J. J.; Adesina, A. S.; Loveridge, E. J.; Tuñón, I.; Moliner, V.; Allemann, R. K. Chemical Ligation and Isotope Labeling to Locate Dynamic Effects during Catalysis by
Dihydrofolate Reductase. Angew. Chem., Int. Ed. 2015, 54, 90169020.

(38) Allemann, R. K.; Evans, R. M.; Tey, L. H.; Maglia, G.; Pang, J.; Rodriguez, R.; Shrimpton, P. J.; Swanwick, R. S. Protein Motions During Catalysis by Dihydrofolate Reductases. Philos. Trans. R. Soc., B 2006, 361, 1317-1321.

(39) Swanwick, R. S.; Maglia, G.; Tey, L. H.; Allemann, R. K. Coupling of Protein Motions and Hydrogen Transfer During Catalysis by Escherichia Coli Dihydrofolate Reductase. Biochem. J. 2006, 394, 259-65.

(40) Allemann, R. K.; Evans, R. M.; Loveridge, E. J. Probing Coupled Motions in Enzymatic Hydrogen Tunnelling Reactions. Biochem. Soc. Trans. 2009, 37, 349-353.

(41) Loveridge, E. J.; Tey, L. H.; Behiry, E. M.; Dawson, W. M.; Evans, R. M.; Whittaker, S. B.; Gunther, U. L.; Williams, C.; Crump, M. P.; Allemann, R. K. The Role of Large-Scale Motions in Catalysis by Dihydrofolate Reductase. J. Am. Chem. Soc. 2011, 133, 2056120570.

(42) Ruiz-Pernía, J. J.; Behiry, E. M.; Luk, L. Y. P.; Loveridge, E. J.; Tuñón, I.; Moliner, V.; Allemann, R. K. Minimization of Dynamic Effects in the Evolution of Dihydrofolate Reductase. Chem. Sci. 2016, 7, 3248-3255.

(43) Wang, Z.; Singh, P.; Czekster, C. M.; Kohen, A.; Schramm, V. L. Protein Mass-Modulated Effects in the Catalytic Mechanism of Dihydrofolate Reductase: Beyond Promoting Vibrations. J. Am. Chem. Soc. 2014, 136, 8333-8341.

(44) Wang, Z.; Chang, E. P.; Schramm, V. L. Triple Isotope Effects Support Concerted Hydride and Proton Transfer and Promoting Vibrations in Human Heart Lactate Dehydrogenase. J. Am. Chem. Soc. 2016, 138, 15004-15010.

(45) Alhambra, C.; Corchado, J.; Sanchez, M. L.; García-Viloca, M.; Gao, J.; Truhlar, D. G. C anonical Variational Theory for Enzyme Kinetics with the Protein Mean Force and Multidimensional Quantum Mechanical Tunneling Dynamics. Theory and Application to Liver Alcohol Dehydrogenase. J. Phys. Chem. B 2001, 105, 1132611340.

(46) Truhlar, D. G.; Gao, J. L.; García-Viloca, M.; Alhambra, C.; Corchado, J.; Sanchez, M. L.; Poulsen, T. D. Ensemble-Averaged Variational Transition State Theory with Optimized Multidimensional Tunneling for Enzyme Kinetics and Other Condensed-Phase Reactions. Int. J. Quantum Chem. 2004, 100, 1136-1152.

(47) Eyring, H.; Stearn, A. E. The Application of the Theory of Absolute Reaction Rates to Proteins. Chem. Rev. 1939, 24, 253-270.

(48) Keck, J. C. Generalized Diffusion Theory of Nonequilibrium Reaction Rates. J. Chem. Phys. 1967, 46, 4211-4213.

(49) Truhlar, D. G.; Garrett, B. C.; Klippenstein, S. J. Current Status of Transition-State Theory. J. Phys. Chem. 1996, 100, 12771-12800.

(50) Pu, J. Z.; Gao, J. L.; Truhlar, D. G. Multidimensional Tunneling, Recrossing, and the Transmission Coefficient for Enzymatic Reactions. Chem. Rev. 2006, 106, 3140-3169.

(51) Zinovjev, K.; Tuñon, I. Quantifying the Limits of Transition State Theory in Enzymatic Catalysis. Proc. Natl. Acad. Sci. U. S. A. 2017, 114, 12390-12395.

(52) Grote, R. F.; Hynes, J. T. The Stable States Picture of Chemical-Reactions. II. Rate Constants for Condensed and Gas-Phase Reaction Models. J. Chem. Phys. 1980, 73, 2715-2732.

(53) Bas, D. C.; Rogers, D. M.; Jensen, J. H. Very Fast Prediction and Rationalization of $\mathrm{pK}(\mathrm{a})$ Values for Protein-Ligand Complexes. Proteins: Struct., Funct., Genet. 2008, 73, 765-783.

(54) Field, M. J. A Practical Introduction to the Simulation of Molecular Systems; Cambridge University Press, 1999.

(55) Singh, U. C.; Kollman, P. A. A Combined Abinitio QuantumMechanical and Molecular Mechanical Method for Carrying out Simulations on Complex Molecular-Systems - Applications to the $\mathrm{CH}_{3} \mathrm{Cl}+\mathrm{Cl}^{-}$Exchange-Reaction and Gas-Phase Protonation of Polyethers. J. Comput. Chem. 1986, 7, 718-730.

(56) Dewar, M. J. S.; Zoebisch, E. G.; Healy, E. F.; Stewart, J. J. P. The Development and Use of Quantum-Mechanical Molecular- 
Models. 76. AM1:A New General-Purpose Quantum-Mechanical Molecular-Model. J. Am. Chem. Soc. 1985, 107, 3902-3909.

(57) Doron, D.; Major, D. T.; Kohen, A.; Thiel, W.; Wu, X. Hybrid Quantum and Classical Simulations of the DihydrofolateReductase Catalyzed Hydride Transfer Reaction on an Accurate Semi-Empirical Potential Energy Surface. J. Chem. Theory Comput. 2011, 7, 34203437.

(58) Kaminski, G. A.; Friesner, R. A.; Tirado-Rives, J.; Jorgensen, W. L. Evaluation and Reparametrization of the OPLS-AA Force Field for Proteins via Comparison with Accurate Quantum Chemical Calculations on Peptides. J. Phys. Chem. B 2001, 105, 6474-6487.

(59) Jorgensen, W. L. Theoretical Studies of Medium Effects on Conformational Equilibria. J. Phys. Chem. 1983, 87, 5304-5314.

(60) Torrie, G. M.; Valleau, J. P. Monte-Carlo Study of a PhaseSeparating Liquid-Mixture by Umbrella Sampling. J. Chem. Phys. 1977, 66, 1402-1408.

(61) Kumar, S.; Bouzida, D.; Swendsen, R. H.; Kollman, P. A.; Rosenberg, J. M. The Weighted Histogram Analysis Method for FreeEnergy Calculations on Biomolecules. I. The Method. J. Comput. Chem. 1992, 13, 1011-1021.

(62) Loveridge, E. J.; Behiry, E. M.; Guo, J. N.; Allemann, R. K. Evidence that a 'Dynamic Knockout' in Escherichia Coli Dihydrofolate Reductase does not Affect the Chemical Step of Catalysis. Nat. Chem. 2012, 4, 292-297.

(63) Loveridge, E. J.; Rodriguez, R. J.; Swanwick, R. S.; Allemann, R. K. Effect of Dimerization on the Stability and Catalytic Activity of Dihydrofolate Reductase from the Hyperthermophile Thermotoga Maritima. Biochemistry 2009, 48, 5922-5933.

(64) Richard, J. P.; Amyes, T. L.; Goryanova, B.; Zhai, X. Enzyme Architecture: on the Importance of Being in a Protein Cage. Curr. Opin. Chem. Biol. 2014, 21, 1-10.

(65) Mhashal, A. R.; Pshetitsky, Y.; Cheatum, C. M.; Kohen, A.; Major, D. T. Evolutionary Effects on Bound Substrate $\mathrm{pKa}$ in Dihydrofolate Reductase. J. Am. Chem. Soc. 2018, 140, 16650-16660.

(66) Mhashal, A. R.; Vardi-Kilshtain, A.; Kohen, A.; Major, D. T. The Role of the $\mathrm{Met}^{20}$ Loop in the Hydride Transfer in Escherichia Coli Dihydrofolate Reductase. J. Biol. Chem. 2017, 292, 1422914239 . 